\title{
Estratégias de leitura e compreensão textual em universitários
}

\author{
Lucicleide Maria de Cantalice \\ Katya Luciane de Oliveira
}

\section{Resumo}

O objetivo deste estudo foi explorar a compreensão textual em universitários e o uso das estratégias metacognitivas de leitura, considerando a faixa etária dos mesmos. Cento e dez universitários (110) do curso de psicologia de uma universidade privada paulista participaram da investigação. Foram utilizados uma escala de estratégias metacognitivas de leitura e um texto de 250 vocábulos preparado segundo a técnica de Cloze em sua versão tradicional, cuja característica é a omissão de todos os quintos vocábulos do texto. A aplicação foi coletiva naqueles estudantes que consentiram participar. Os dados foram submetidos à estatística inferencial e evidenciaram relação estatisticamente significativa entre a pontuação na escala de estratégias metacognitivas de leitura e os escores do Cloze. Foi possível também evidenciar diferenças na pontuação na compreensão em leitura dos estudantes, considerando a faixa etária dos mesmos.

Palavras-chave: Leitura, Teste de Cloze, estudantes universitários.

\section{Reading strategies and textual comprehension among university students}

\begin{abstract}
In this study we explore the reading and understanding of texts by university students. We used the metacognitive reading strategies considering students' age. We work with one hundred and ten students from Psychology course at a private University located in the State of São Paulo. We apply reading metacognitive strategy scale and a text containing 250 words prepared according to Cloze technique, which is characterized by omitting every fifth word. The work was developed with the students who accepted to take part in the investigation. The data were submitted to inferential statistics and revealed the relation existing between the punctuation of reading metacognitive strategies scale and the Cloze's scores. It was also possible to evidence the differences in the university students' reading comprehension according to their ages.
\end{abstract}

Key-words: Reading, Cloze testing, College Students.

\section{Estrategias de lectura y comprensión textual en estudiantes universitarios}

\section{Resumen}

El objetivo de este estudio fue explorar la comprensión textual en universitarios y el uso de las estrategias metacognitivas de lectura, considerando la franja etaria de los mismos. Ciento diez universitarios (110) de la facultad de Psicología de una universidad privada paulista participaron de la investigación. Una escala de estrategias metacognitivas de lectura y un texto de 250 vocablos preparados según la técnica de Cloze, en su versión tradicional, fueron utilizados, cuya característica es la omisión de todos los quintos vocablos del texto. La aplicación fue colectiva en aquellos estudiantes que consintieron participar. Los datos fueron sometidos a la estadística inferencial y evidenciaron relación estadísticamente significativa entre los puntajes en la escala de estrategias metacognitivas de lectura y el puntaje de Cloze. Fue posible también evidenciar diferencias en el puntaje de la comprensión de lectura de los estudiantes, considerando la franja etaria de los mismos.

Palabras-clave: Lectura, test de Cloze, Estudiantes universitários. 


\section{Introdução}

\section{Compreensão textual}

A leitura é um processo cada vez mais presente no dia a dia do ser humano, possibilitando uma melhor inserção social. Ela envolve praticamente quase todos os aspectos da psicologia humana: capacidade sensorial, percepção, aprendizagem, motivação, pensamento, memória, dentre outros. Tal processo inicia-se antes mesmo do ingresso do indivíduo em instituições de ensino, culminando na última etapa de escolarização formal, a universidade.

A leitura e sua compreensão é considerada uma habilidade dinâmica que envolve criatividade, flexibilidade, ritmo e fluência. Quando um leitor exibe esses comportamentos, certamente demonstrará uma maior capacidade de fazer críticas e criar analogias com outras informações lidas. Portanto, no ensino superior, a habilidade de compreensão textual é de suma importância para uma formação de qualidade (Oliveira \& Santos, 2005; Oliveira, Suehiro, \& Santos, 2004).

Mesmo se constituindo numa habilidade tão importante, diversas pesquisas realizadas com universitários (Centofanti, Ferreira, \& Del Tedesco, 1997; Oliveira, Santos, \& Primi, 2003; Oliveira \& Santos, 2005; Oliveira \& Santos, 2006; Santos, 1991; Santos, Primi, Taxa, \& Vendramini, 2002; Suehiro, Santos, \& Oliveira, 2004) apontam que essa população apresenta sérias limitações na compreensão em leitura. Esse fato interfere de forma direta e negativa no desempenho acadêmico.

Maki, Shields, Wheeler e Zacchilli (2005), Oliveira e Santos (2006) e Oliveira, Santos e Boruchovitch (2007) argumentam que a baixa compreensão textual é produto de um repertório pouco diversificado de conhecimentos prévios, o que possivelmente é resultado de uma escolarização falha que apresenta uma série de lacunas. Esse fato é lastimável, visto que uma formação completa exige a compreensão textual necessária para entender os conteúdos técnicos exigidos nesse nível de escolaridade.

Com a perspectiva de se levantar a compreensão textual de estudantes universitários e possíveis implicações no desempenho, alguns estudiosos (Oliveira \& Santos, 2006; Richardson, 1994; Silva, \& Santos, 2004; Simonite, 1997; Trueman \& Hartley, 1996) buscaram averiguar eventuais diferenças entre universitários de diferentes faixas etárias. Nessa perspectiva, Trueman e Hartley (1996) denominaram os estudantes mais jovens (17 a 21 anos) como tradicionais. $\mathrm{Na}$ segunda faixa, os estudantes intermediários (22 a 25 anos). Por fim, na terceira faixa encontram-se os estudantes maduros (26 anos ou mais). Os estudos desenvolvidos com essa finalidade indicam que há diferença entre os estudantes mais jovens e os mais maduros em diferentes habilidades acadêmicas.

Embora na literatura internacional estejam crescendo as investigações que visam analisar o desempenho em diversas competências, considerando a variável faixa etária, tais estudos ainda são restritos no Brasil. De forma geral, essa variável tem sido analisada apenas secundariamente. Nessa direção, aventa-se a hipótese de que a habilidade de compreensão textual, bem como o emprego de estratégias de leitura no momento do estudo, podem se diferenciar em razão da faixa etária dos estudantes.

Sobre esse aspecto, Singer e Donlan (1982) discutem que a leitura com compreensão consiste na interação entre recursos dos leitores e as características do texto. Para que esta interação ocorra, há necessidade do leitor utilizar conhecimentos prévios e estruturas de conhecimento preexistentes que se assemelham com a informação contida no texto. Assim, essa interação envolve a utilização de estratégias de leitura, que venham auxiliar o leitor na aquisição do conhecimento.

\section{Estratégias de leitura}

Pode-se conceituar estratégias como métodos de abordagem de um problema ou tarefa e modos de atuação para alcançar um determinado objetivo. As estratégias de leitura, especificamente, caracterizam-se por serem planos flexíveis que os leitores usam, adaptados aos diferentes tipos de textos. Pellegrini (1996) destaca que as estratégias de leitura variam de acordo com o material a ser lido e a abordagem ou o plano elaborado previamente pelo leitor para facilitar a sua compreensão.

As estratégias de leitura podem ser cognitivas ou metacognitivas. As primeiras podem ser caracterizadas como comportamentos e pensamentos que interferem na aquisição da informação lida, ou ainda, como formas deliberadas de decodificação dos símbolos acerca da linguagem, constituindo a construção de significado (Kletzien, 1991; Kopke, 1997). Já as metacognitivas são definidas como a consciência que o leitor apresenta sobre o próprio nível de compreensão durante a leitura e a habilidade para controlar as ações cognitivas por meio de estratégias que facilitem a compreensão de um determinado tipo de texto ou de tarefa determinada (Bolívar, 2002). No processo de metacognição, o leitor é capaz de planejar, monitorar e regular o seu próprio pensamento antes, durante e após a leitura.

Numa revisão de literatura sobre o tema, Mokhtari e Reichard (2002) constatam que a consciência e o monitoramento do processo de compreensão são aspectos importantes para a habilidade em leitura, uma vez que a avaliação metacognitiva do processo cognitivo e motivacional de uma leitura tem liderado as pesquisas na área de compreensão em leitura. O processo de consciência e de monitoramento são aspectos semelhantes e envolvem o autocontrole quando monitoram e regulam a compreensão de um texto.

A utilização de estratégias de leitura compreende três momentos: o antes, o durante e o após a leitura. $\mathrm{Na}$ pré-leitura, é feita uma análise global do texto (do título, dos tópicos e das figuras/gráficos) e também predições e o uso do conhecimento prévio. Durante a leitura, é feita uma compreensão da mensagem passada pelo texto, uma seleção das informações relevantes, uma relação entre 
as informações apresentadas no texto e uma análise das predições feitas antes da leitura, para confirmá-las ou refutá-las. Depois da leitura, é feita uma análise com o objetivo de rever e refletir sobre o conteúdo lido, ou seja, sobre a importância da leitura, o significado da mensagem, a aplicação para solucionar problemas e a verificação de diferentes perspectivas apresentadas para o tema. Também é realizada uma discussão da leitura, com expressão e comunicação do conteúdo lido após análise e reflexão, seguidas de um resumo e de uma releitura do texto (Duke \& Pearson, 2002; Kopke, 1997).

Mokthari e Reichard (2002) conceituam metacognição como o conhecimento sobre estado e habilidade cognitivas. Nessa mesma perspectiva, Kopke (2001) afirma que a metacognição pressupõe reflexão e controle consciente sobre o próprio fazer, sobre a própria capacidade de entender o conhecimento e controle voluntário acerca dos próprios processos cognitivos, e envolve principalmente o automonitoramento como favorecedor da compreensão.

Kopke (2001) descreve como primeiro aspecto da metacognição o conhecimento dos próprios processos cognitivos sobre si mesmo (pontos fortes, pontos fracos, preferências pessoais); sobre a tarefa (níveis de dificuldade, demandas) e sobre o uso de estratégias (quais, quando, por que e para que). E, como segundo aspecto, a regulação e o controle do comportamento, considerando-se a existência de três tipos de controle: planejamento, que envolve a organização de uma sequência de atividades, que são apropriadas para a leitura; monitoramento, que se refere à capacidade do sujeito de supervisionar o seu próprio processo de leitura; e regulação, que auxilia o aluno a modificar seu comportamento de estudo e permite que melhore suas dificuldades de compreensão.

Shraw (1998) esclarece que as estratégias metacognitivas de leitura também podem receber classificação mediante características próprias. Entre essas características, encontram-se as globais (utilizadas para uma análise geral do texto); as de suporte à leitura (relacionadas ao uso de materiais de apoio, como referência, anotações, dentre outros) e as de solução de problemas (usadas pelo leitor à medida que este se encontra com dificuldades de compreensão).

Estudos internacionais (Brown, Pressley, Van Meter, \& Schuder, 1996; Lorch, Lorch, \& Klusewrtz, 1993; Magliano, Graesser, \& Trabasso, 1999; Mokhtari \& Reichard, 2002; Schmidt, 2000; Song, 1998) foram realizados visando explorar o emprego das estratégias de leitura e seus efeitos em uma leitura com maior compreensão textual. Em âmbito nacional, alguns estudos (Cantalice, 2004; Dias, Morais, \& Oliveira, 1995; Ferreira \& Dias, 2002; Kopke, 2001) também foram desenvolvidos com essa finalidade. Assim, de um modo geral, as pesquisas estrangeiras e nacionais indicam que os estudantes recorrem às estratégias de leitura no momento do estudo e que o emprego dessas estratégias contribui para uma melhora na compreensão textual.

A utilização das estratégias de leitura considerando a faixa etária dos estudantes não tem despertado o interesse dos pesquisadores. Em uma busca acerca dos estudos realizados sobre o tema, destaca-se a investigação de
Morles, Amat, Donis e Urquhart (1997) que, ao comparar universitários com alto e baixo rendimento em leitura, verificaram diferenças entre as estratégias de leitura utilizadas por estudantes para lidar com as dificuldades de um texto, ressaltando a importância de se conhecer os problemas e as estratégias mais frequentemente empregadas, bem como sua eficácia na compreensão da leitura. A amostra foi composta por 14 sujeitos, sendo sete novatos e sete experientes, e foi utilizado um texto autêntico com 277 palavras. Os resultados apontaram que os leitores novatos encontraram, com maior frequência, problemas relacionados à integração da informação com o desconhecimento do vocabulário, além de não saberem resolver os problemas que surgiam. Os leitores mais experientes tinham poucos problemas com a integração entre as informações e, quando desconheciam o significado de algumas palavras, eram capazes de resolver este problema e compreender a leitura. Os leitores novatos parecem estar menos conscientes que os experientes tanto em relação a sua dificuldade de vocabulário quanto ao seu nível de compreensão. Quanto às estratégias empregadas para resolver problemas de compreensão, os novatos preferem a releitura e os experientes, estratégias específicas do tipo solução de problemas para cada dificuldade. Verificouse que os leitores experientes usam um maior número de estratégias de leitura que os novatos.

Rhoder (2002) observa que se pode ensinar alunos a usarem estratégias para ler e entenderem o texto, de forma implícita e explícita. Contudo, para os alunos compreenderem um texto e usarem estratégias de leitura, necessitam conhecer quais estratégias podem usar, como, quando, onde e por que usá-las. Para que isso ocorra, é necessário um suporte no qual aprendam as estratégias em situações autênticas de modo a aprimorar também a compreensão textual.

Com base nas considerações trazidas e tendo em vista que a universidade é a última etapa de escolarização formal, o presente estudo buscou explorar a compreensão textual em estudantes universitários e o uso das estratégias metacognitivas de leitura, considerando a faixa etária dos mesmos.

\section{Método}

\section{Participantes}

O estudo contou com 110 estudantes universitários do curso de psicologia de uma universidade privada paulista. A média de idade dos estudantes foi de 24 anos e oito meses $(D p=5,7)$, a mínima, 19 anos e a máxima, 48 . O gênero feminino representou $82,7 \%(n=91)$ da amostra e o masculino, $17,3 \%(n=19)$.

\section{Instrumentos}

Utilizou-se um texto de autoria de Luís Fernando Veríssimo (1995) intitulado "Desentendimento" preparado conforme os parâmetros da técnica de Cloze (Taylor, 1953) 
em que se emprega um texto de aproximadamente 250 vocábulos do qual se omitem todos os $5^{\circ} \mathrm{s}$ vocábulos e no local coloca-se um traço de tamanho proporcional ao da palavra omitida. A correção empregada foi a literal, que aceitou como acerto o preenchimento exato da palavra omitida. Atribuiu-se 1 ponto para os acertos e 0 para os erros, portanto a pontuação poderia variar de 0 a 46 pontos.

Para a classificação das respostas do teste de Cloze, adotaram-se três critérios estabelecidos por Bormuth (1968). O primeiro refere-se ao nível de frustração e diz respeito ao fato do leitor não ter abstraído de forma satisfatória a informação lida (pontuação de até $44 \%$ de acertos). O segundo critério é o instrucional, compreensão apenas suficiente para o entendimento (de $44,1 \%$ a $57 \%$ de acertos). Por fim, o nível independente indica uma compreensão criativa, autônoma e fluente do texto (superiores a $57 \%$ de acertos).

Foi utilizada a Escala de Estratégias Metacognitivas de Leitura - formato Universitário (EMeL-U) (Joly, 2004). A escala tem por objetivo avaliar o tipo e a frequência de estratégias de leitura que os graduandos utilizam antes, durante e depois da leitura de textos acadêmicos. É composta por 35 afirmações do tipo likert com cinco opções de frequência: nunca, poucas vezes, algumas vezes, muitas vezes e sempre. A escala é dividida em três fatores. O primeiro abarca 18 estratégias metacognitivas globais (Fator 1), o segundo, 6 estratégias metacognitivas de suporte à leitura (Fator 2) e o último fator diz respeito a 11 estratégias metacognitivas de solução de problemas (Fator 3). Esses fatores explicam $42,12 \%$ da variabilidade da escala. A precisão total da escala é de $\alpha=0,73$.

No que se refere à pontuação, a opção nunca recebe a pontuação 1 ; respostas assinaladas poucas vezes recebem a pontuação 2; algumas vezes, 3; muitas vezes, 4; e sempre, 5 . Desse modo, as pontuações podem variar de 0 a 175 na escala geral, de 0 a 90 no fator 1 , de 0 a 30 no fator 2 e de 0 a 55 no fator 3 .

\section{Procedimento}

Inicialmente os objetivos do estudo foram expostos. Os instrumentos foram respondidos pelos estudantes que consentiram na participação por meio da assinatura do Termo de Consentimento Livre e Esclarecido. A aplicação foi coletiva, em sessão única. A escala de estratégias metacognitivas de leitura foi aplicada num primeiro momento e, em seguida, ocorreu a aplicação do teste de Cloze. O tempo de aplicação foi de aproximadamente 60 minutos, considerando os dois instrumentos. Acrescenta-se que esta pesquisa foi baseada e está em consonância com a Resolução 196/96 do Conselho Nacional de Saúde.

\section{Resultados}

Os dados foram organizados em planilha e submetidos à análise estatística descritiva e inferencial, visando atender aos objetivos deste estudo. Primeiramente, os estudantes foram agrupados em faixas etárias. Cabe esclarecer que, embora Trueman e Hartley (1996) tenham classificado os universitários em três faixas etárias: tradicionais (17 a 21 anos), intermediários (22 a 25 anos) e maduros (26 anos ou mais), a presente pesquisa dividiu os estudantes em duas faixas etárias visto que uma análise preliminar dos dados indicou diferença no agrupamento realizado neste estudo. Assim, a primeira faixa contemplou as idades de 19 a 25 anos $(72,7 \%, n=80)$ e a segunda, as idades de 26 a 48 anos $(25,5 \%, n=28)$. Em acréscimo, duas $(1,8 \%)$ pessoas não informaram a idade.

No que se refere ao Teste de Cloze, observou-se que a média de acertos foi de 22,0 ( $D p=4,4)$, a pontuação mínima, 11 pontos e a máxima, 31. Quanto à classificação, segundo os critérios estabelecidos por Bormuth (1968), evidenciouse que $33 \% \quad(n=30)$ dos estudantes estavam no nível classificado como Frustração, 56,4\% (n=62) demonstraram uma compreensão Instrucional e 13,6\% ( $n=15)$ obtiveram uma compreensão Independente. Na Tabela 1, pode-se observar os percentuais de classificação de Bormuth (1968), considerando a faixa etária do estudante.

Tabela 1. Frequência e porcentagem das classificações do nível de compreensão, considerando a faixa etária do estudante.

\begin{tabular}{|l|c|c|}
\hline Classificações da compreensão & $\begin{array}{c}\text { Faixa 1 } \\
F \text { e \% }\end{array}$ & $\begin{array}{c}\text { Faixa 2 } \\
F \text { e \% }\end{array}$ \\
\hline Frustração & 18 & 13 \\
\hline Instrucional & 22,5 & 46,4 \\
\hline
\end{tabular}

Fonte: Elaboração dos autores

Em seguida, foi realizado um levantamento das pontuações obtidas na escala de estratégias metacognitivas de leitura escala geral e nos respectivos fatores. A Tabela 2 apresenta os dados de média, desvio-padrão, pontuação mínima e máxima.

Verificaram-se as pontuações obtidas na escala de estratégias metacognitivas de leitura escala geral e nos respectivos fatores, considerando a faixa etária dos participantes. Na Tabela 3, os dados de distribuição da pontuação na escala geral e nos três fatores da escala, considerando a faixa etária dos participantes.

$O$ teste $U$ de Mann-Whitney foi utilizado para buscar possíveis diferenças na escala de estratégias de leitura e respectivos fatores e os escores do Cloze. Os dados indicaram diferença estatisticamente significativa entre os estudantes mais maduros e aqueles mais jovens somente na pontuação do teste de Cloze, tendo em vista $U=711,500$; $p=0,004$. No que tange à escala de estratégias de leitura e seus fatores, a diferença entre os estudantes mais jovens e 
Tabela 2. Distribuição da média, desvio-padrão, pontuação mínima e máxima na escala de estratégias metacognitivas de leitura.

\begin{tabular}{|l|c|c|c|c|}
\hline Escala & $M$ & $D p$ & $\begin{array}{c}\text { Pontuação } \\
\text { Mínima }\end{array}$ & $\begin{array}{c}\text { Pontuação } \\
\text { Máxima }\end{array}$ \\
\hline Fator 1 - metacognitivas globais & 61,6 & 9,1 & 37 & 85 \\
\hline Fator 2 - suporte à leitura & 21,2 & 5,0 & 7 & 30 \\
\hline Fator 3 - solução de problemas & 45,4 & 6,0 & 29 & 57 \\
\hline
\end{tabular}

Fonte: Elaboração dos autores

Tabela 3. Pontuação na escala geral e nos três fatores da escala, considerando a faixa etária dos participantes.

\begin{tabular}{|l|c|c|}
\hline Escala & Faixa 1 & Faixa 2 \\
& M e Dp & M e Dp \\
\hline Fator 1 - metacognitivas globais & 61,7 & 61,1 \\
& 8,9 & 10,1 \\
\hline Fator 2 - suporte à leitura & 21,3 & 21,1 \\
\hline \multirow{2}{*}{ Fator 3 - solução de problemas } & 5,3 & 4,2 \\
\hline
\end{tabular}

Fonte: Elaboração dos autores

mais maduros não foi estatisticamente significativa. A Figura 1 apresenta a distribuição dos Ranks das pontuações dos participantes no Cloze.

Para avaliar se havia relação entre as pontuações na escala de estratégias metacognitivas de leitura e os escores do Cloze, utilizou-se a correlação de Spearman. Os dados demonstraram relação estatisticamente significativa e positiva entre o fator estratégias metacognitivas de suporte à leitura e a pontuação geral da escala de estratégias metacognitvas de leitura e a pontuação no Cloze. A Tabela 4 demonstra os coeficientes de correlação obtidos.

\section{Discussão e Conclusão}

No que diz respeito à compreensão textual, a média $(M=22)$ de acertos do Teste de Cloze ficou muito aquém do esperado para o nível de compreensão que estudantes universitários devem exibir. Os estudantes não acertaram nem a metade de acertos possíveis no teste de Cloze, isto é, 23 pontos. Nenhum estudante atingiu o total de pontos possíveis (46 pontos) visto que a pontuação máxima foi 31 pontos.

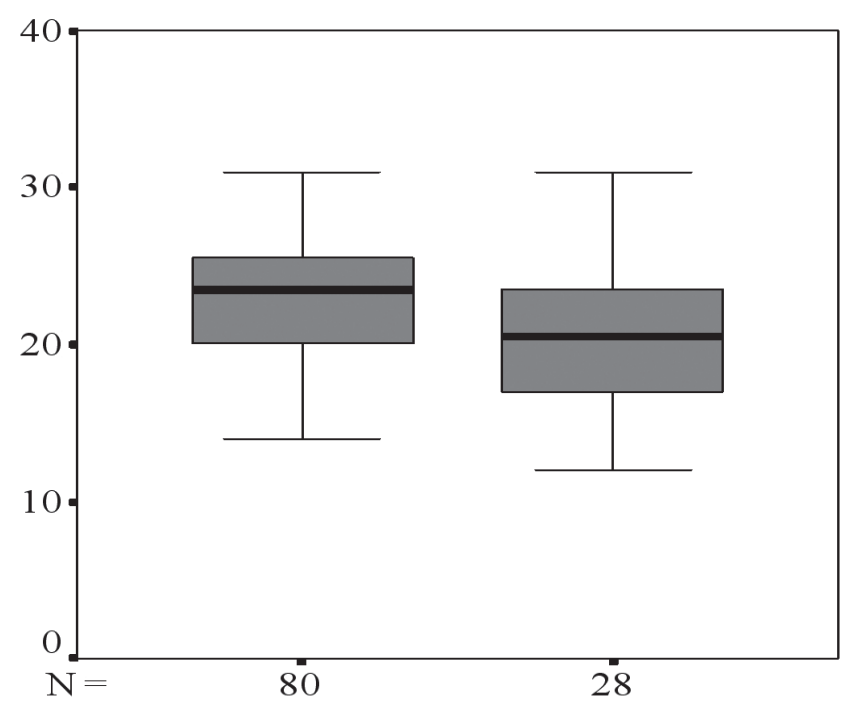

Figura 1. Box-plot dos participantes do Rank de médias, considerando as faixas etárias dos estudantes.

Fonte: Elaboração dos autores 
Tabela 4. Distribuição dos coeficientes de correlação e nível de significância entre a pontuação geral da escala de estratégias de estratégias metacognitivas de leitura e fatores e os escores do Cloze.

\begin{tabular}{|l|c|c|}
\hline & \multicolumn{2}{|c|}{ Teste Cloze } \\
\hline & $r_{s}$ & $p$ \\
\hline Fator 1 - metacognitivas globais & 0,175 & 0,074 \\
\hline Fator 2 - suporte à leitura & 0,142 & 0,148 \\
\hline Fator 3 - solução de problemas & 0,234 & 0,016 \\
\hline Pontuação Geral & 0,227 & 0,020 \\
\hline
\end{tabular}

Fonte: Elaboração dos autores

Desse modo, ao se considerar os critérios estabelecidos por Bormuth (1968), pode-se afirmar que parte considerável da amostra (33\%) está no nível de Frustração de compreensão, o que denota estudantes que não conseguem abstrair de forma satisfatória a informação lida. A maior parte $(56,4 \%)$ foi classificada no nível Instrucional, isto é, conseguem compreender de forma suficiente o conteúdo lido, contudo não exibem comportamento criativo, flexível, autônomo e fluente, o que caracterizaria o nível independente, tipo de compreensão que apenas uma pequena parte $(13,6 \%)$ da amostra apresentou.

Os dados obtidos na presente pesquisa corroboram os estudos realizados anteriormente (Centofanti e cols., 1997; Oliveira e cols., 2003; Oliveira \& Santos, 2005; Oliveira \& Santos, 2006; Santos, 1991; Santos e cols., 2002; Suehiro e cols., 2004). Esses dados são preocupantes uma vez que se tratam de universitários que futuramente ingressarão no mercado de trabalho e, se não houver programas interventivos visando remediar a situação, presumivelmente sairão com uma qualificação técnica insuficiente para os desafios inerentes à profissão.

$O$ teste $U$ de Mann-Whitney evidenciou que os estudantes mais jovens apresentaram uma maior compreensão em leitura do que os mais maduros, conforme consta na Figura 1. Uma análise qualitativa da Tabela 1 indica que apenas um estudante mais maduro conseguiu exibir um nível de compreensão Independente. Esses dados podem sugerir que os estudantes mais novos têm uma maior abstração no que tange à compreensão da informação lida. Deve-se também considerar que os estudantes mais jovens também tiveram um maior percentual de classificação em compreensão Independente. Esses resultados apontam que, de fato, há uma diferença entre os estudantes mais jovens e aqueles mais maduros, conforme já foi constatado anteriormente (Oliveira \& Santos, 2006; Richardson, 1994; Silva \& Santos, 2004; Simonite, 1997; Trueman \& Hartley, 1996). Contudo, os dados obtidos neste estudo devem ser vistos com ressalvas pois há que se ponderar que a amostra poderia ter sido maior e mais diversificada. Ainda assim, com o mérito de se explorar diferenças por faixa etária, o presente estudo atendeu aos propósitos estabelecidos nos objetivos.

Quanto às estratégias de leitura, considera-se que a média $(M=128,3)$ da pontuação geral na escala foi positiva visto que a pontuação máxima poderia chegar a 175. No que tange ao uso das estratégias de leitura globais, a pontuação poderia chegar a 90 pontos e a média foi de 61,6; para as estratégias de suporte à leitura, a média foi de 21,2 e a pontuação poderia chegar a 30 pontos e, por fim, no emprego de estratégias de solução de problemas, a média de pontos foi de 45,4 e a pontuação poderia chegar a 55 pontos. Assim, ao que parece, os participantes demonstraram que recorrem às estratégias no momento do estudo. Novamente, os dados obtidos na presente pesquisa corroboram os estudos realizados anteriormente (Brown e cols., 1996; Cantalice, 2004; Dias e cols., 1995; Ferreira \& Dias, 2002, Kopke, 2001; Lorch e cols., 1993; Magliano e cols., 1999; Mokhtari \& Reichard, 2002; Schmidt, 2000; Song, 1998). Cabe, contudo, questionar se os alunos sabem quando e quais estratégias empregar de modo a aquilatar cada vez mais seu estudo.

A análise das pontuações na escala de estratégias de leitura e respectivos fatores, tendo em vista a faixa etária, indicou que as faixas não se diferenciaram. Ao se analisar a Tabela 3, é possível observar que as pontuações entre as faixas ficaram muito próximas, assim sendo, o teste $U$ de Mann-Whitney não levantou diferença estatisticamente significativa entre as faixas na escala de estratégias de leitura e seus fatores. Como não houve diferença entre as faixas, esses dados não confirmam os resultados obtidos por Morles e cols. (1997) que indicaram que os estudantes mais maduros recorrem mais às estratégias de leitura em relação aos mais jovens. Portanto, visando ampliar as considerações sobre o emprego de estratégias de leitura e eventuais diferenças por faixa etária, seria ingênuo não ressaltar a necessidade de que novos estudos sejam realizados.

Com o tratamento de correlação de Spearman, foi possível aferir relação entre as pontuações na escala de estratégias de leitura e os escores do Teste de Cloze. Relações estatisticamente significativas (Tabela 4) foram observadas entre o fator 3 - solução de problemas - e a pontuação geral da escala e os escores do Cloze. Pode-se aventar com tais resultados que, quanto melhor a compreensão textual, maior é a incidência de uso das estratégias de leitura $\left(r_{s}=0,227\right.$; $p=0,020$ ). Como os universitários apresentaram uma baixa compreensão textual, sugere-se, ainda, que as estratégias de solução de problemas são empregadas com bastante frequência à medida que os estudantes encontram grande dificuldade na compreensão $\left(r_{s}=0,234 ; p=0,016\right)$.

As evidências reveladas no presente estudo apontam a necessidade de que programas interventivos sejam implementados de modo que o emprego e a diversificação no uso das estratégias de leitura sejam incentivados no ensino superior. Desse modo, poder-se-ia melhorar a compreensão em leitura e, consequentemente, a qualidade técnica profissional. 


\section{Considerações Finais}

Tendo em vista os resultados obtidos, verifica-se a importância de se desenvolver pesquisas e intervenções com o leitor universitário. A universidade como última etapa de escolarização formal espera do graduando um nível de leitura independente, por meio da qual seja possível abstrair, compreender e analisar de forma crítica e criativa o conhecimento adquirido.

Todavia, a literatura destaca os estudos realizados cujos resultados revelam o baixo nível de compreensão e a pouca utilização de estratégias de leitura dos universitários. Essas considerações podem ser justificadas devido à da ausência de programas de intervenção em níveis escolares anteriores à universidade.

O fato das estratégias de leitura de solução de problemas serem as mais utilizadas revela que grande parte dos leitores busca o uso deste recurso somente quando encontra dificuldade na compreensão, fato lamentável. Chama atenção, entretanto, que novos estudos devem ser realizados, visando ampliar as considerações trazidas na presente pesquisa. Além disso, nota-se uma crescente preocupação com as variáveis envolvidas neste estudo (compreensão textual e estratégias de leitura). Cada vez mais os estudos têm apontado a necessidade da implementação de programas interventivos que auxiliem os estudantes a saberem quais momentos e quais estratégias devem utilizar no momento do estudo. Portanto, cabe à universidade, instituição voltada ao ensino e à pesquisa, viabilizar aos seus educandos a possibilidade de desenvolvimento de habilidades que lhes propiciem um melhor entendimento textual, o que permitirá uma melhor compreensão da realidade que os cerca.

\section{Referências}

Bolívar, C. R. (2002). Mediación de Estrategias Metacognitivas en Tareas Divergentes Y Transferencia Recíproca. Investigación Y Postgrado, 17(2), 1-20.

Bormuth, J. R. (1968). Cloze test readability: criterion reference scores. Journal of Educational Measurement, 5, 189-196.

Brown, R., Pressley, M., Van Meter, P., \& Shuder, T. (1996). A Quasiexperimental Validation of Transactional Strategies Instrucion with Low-achievinh Second-grade Readers. Journal of Educational Psychology, 88, 18-37.

Cantalice, L. M. (2004). Ensino de estratégias de leitura. Psicologia Escolar e Educacional, 8, 105-106.

Centofanti, E. M., Ferreira, S. M., \& Del Tedesco, T. (1997). Compreensão da leitura por universitários de psicologia. Em G. P. Witter (Org.), Leitura e universidade (pp. 33-60). Campinas: Alínea.

Conselho Nacional de Saúde.(1996). Resolução 196/96. Diretrizes e Normas Regulamentadoras de Pesquisa. Recuperado: 19 out. 2005. Disponível: http://www.conselho.saude.gov.br.
Dias, M. G. B. B., Morais, E. P. M., \& Oliveira, M. C. N. P. (1995). Dificuldades na Compreensão de Textos: Uma Tentativa de Remediação. Arquivos Brasileiros de Psicologia, 47(4), 13-24.

Duke. N. K., \& Pearson, P. D. (2002). Effective Practices for Developing Reading Comprehension. Em A. E. Farstrup \& S. J. Samuels (Orgs.), What Research Has to Say About Reading Instruction (pp. 205-242). Newark, Del: Internacional Reading Association.

Ferreira, S. P. A., \& Dias, M. G. B. B. (2002). Compreensão de Leitura: Estratégias de Tomar Notas e da Imagem Mental. Psicologia Teoria e Pesquisa, 18(1), 51-62.

Joly, M. C. A. (2004). Escala de Estratégias Metacognitivas de Leitura - formato Universitário (EMeL-U). Manuscrito não publicado do Programa de Pós-graduação Stricto Sensu em Psicologia da Universidade São Francisco.

Kletzien, S. B. (1991). Strategy used by Good and Poor Comprehenders Reading Expository Text of Differing Levels. Reading Research Quarterly, 26(1), 67-83.

Kopke, H. F. (1997). Estratégias para Desenvolver a Metacognição e a Compreensão de Textos Teóricos na Universidade. Psicologia Escolar e Educacional, 1(2-3), 59-67.

Kopke, H. F. (2001). Estratégias em Compreensão da leitura: Conhecimento e Uso por Professores de Língua Portuguesa. Dissertação de Mestrado, Universidade de São Paulo, São Paulo.

Lorch, R. F., Lorch, E. P., \& Klusewitz, M. A. (1993). College Student's Conditional Knowledge About Reading. Journal of Educational Psychology, 85(2), 239-252.

Magliano, J. P., Graesser, A., \& Trabasso, T. (1999). Strategic Processing During Comprehension. Journal of Educacional Psychology, 9(4), 615-629.

Maki, R. H., Schields, M., Wheeler, A. E., \& Zacchini, T. L. (2005). Individual differences in absolute and relative metacomprehension accurancy. Journal of Educational Psychology, 97(4), 723-731.

Mokhtari, K., \& Reichard, C. A. (2002). Assessing Students' Metacognitive Awareness of Reading Strategies. Journal of Educational Psychology, 94(2), 249-259.

Morles, A., Amat, M., Donis, Y., \& Urquhart, R., (1997). Resolución de Problemas de Procesamiento de La Información durante La Lectura. Lectura Y Vida, 18(3), 13-22.

Oliveira, K. L., Santos, A. A. A., \& Primi, R. (2003). Estudo das relações entre compreensão em leitura e desempenho em disciplinas na universidade. Interação em Psicologia, 7(1), 1925.

Oliveira, K. L., \& Santos, A. A. A. (2005). Compreensão em leitura e avaliação da aprendizagem em universitários. Psicologia: Reflexão e Crítica, 18(1), 118-124.

Oliveira, K. L., \& Santos, A. A. A. (2006). Compreensão de textos e desempenho acadêmico. Psic - Revista de Psicologia da Vetor Editora, 7(1), 19-27.

Oliveira, K. L., Santos, A. A. A., \& Boruchovitch, E. (2007). Estudo de intervenção para a compreensão em leitura e produção de textos na universidade. Manuscrito submetido à Psicologia em Pesquisa da Universidade Federal de Juiz de Fora.

Oliveira, K. L., Suehiro, A. C. B., \& Santos, A. A. A. (2004). Avaliação da aprendizagem no ensino superior: estudo da relação com a compreensão em leitura. Em C. Machado, L. S. Almeida, M. Gonçalves \& V. Ramalho (Orgs.), Avaliação Psicológica: Formas e Contextos (pp.217-223). Braga, Portugal: Psiquilíbrios Edições. 
Pellegrini, M. C. K. (1996). Avaliação dos Níveis de Compreensão e Atitudes frente à Leitura em Universitários. Dissertação de Mestrado, Universidade São Francisco. Bragança Paulista, São Paulo.

Rhoder, C. (2002). Mindful Reading: Strategy Training that Facilitates Transfer. Journal of Adolescent \& Adult Literacy, 45(6), 498-512.

Richardson, J. T. E. (1994). Mature students in higher education: I. A literatura survey on approaches to studying. Studies in Higher Education, 19(3), 309-325.

Santos, A. A. A. (1991). Desempenho em leitura: um estudo diagnóstico da compreensão e hábitos de leitura entre universitário. Estudos de Psicologia, 8(1), 6-19.

Santos, A. A. A., Primi, R., Taxa, F., \& Vendramini, C. M. M. (2002). O teste de Cloze na avaliação da compreensão em leitura. Psicologia: Reflexão e Crítica, 15(3), 549-560.

Schmidt, M. C. (2000). A Questionnaire to Measure Children's Awareness of Strategic Reading Process. Em S. J. Barrentine (Org.), Reading Assessment (pp.189-198). Newark, Del: IRA.

Schraw, G. (1998). Promoting general metacognitive awareness. Instructional Science, 26, 113-125.

Silva, M. J. M., \& Santos, A. A. A. (2004). A avaliação da compreensão em leitura e o desempenho acadêmico de universitários. Psicologia em Estudo, 9(3), 459-457.
Simonite, V. (1997). Academic achievement of mature students on a modular degree course. Journal of Further and Higher Education, 21(2), 241-249.

Singer, H., \& Donlan, D. (1982). Problem-Solving Schema With Question Generation for Comprehensiion of Complex Short Stories. Em R. B. Ruddell, M. R. Ruddell \& H. Singer (Orgs.), Theorical Models and Processes of Reading (pp.166-186). Newark, Del: IRA.

Song, M. (1998). Teaching Reading Strategies in an Ongoing EFL University Reading Classroom. Journal of English language teaching, 8, 41-54.

Suehiro, A. C. B., Santos, A. A. A., \& Oliveira, K. L. (2004). Habilidades em compreensão da leitura: um estudo com alunos de psicologia. Estudos de Psicologia, 21(2), 29-41.

Taylor, W. L. (1953). Cloze procedure: a new tool for measuring read-ability. Journalism Quarterly, 30, 415-433.

Trueman, M., \& Hartley, J. (1996). A comparison between the timemanagement skills and academic performance of mature and traditional-entry university students. University of Keele, 199215.

Veríssimo, L. F. (1995). Desentendimento. Ícaro Revista de bordo da Varig, (136), Ano XII.

\section{Sobre as Autoras}

Lucicleide Maria de Cantalice (lucicleide.Cantalice@saofrancisco.edu.br) Universidade São Francisco.

\section{Katya Luciane de Oliveira}

Universidade São Francisco.

\section{Correspondência}

Lucicleide Maria de Cantalice

Universidade São Francisco

R: Alexandre Rodrigues Barbosa, 45 - Centro. Itatiba /SP. CEP.13.251-900

Recebido em: 27/02/2008

Aprovado em: 09/12/2009 